\title{
SATELLITE IMAGE ANALYSIS USING NEURAL NETWORKS
}

\author{
Roger A. Sheldon \\ Ford Aerospace \\ Seabrook, MD
}

\begin{abstract}
The tremendous backlog of unanalyzed satellite data necessitates the development of improved methods for data cataloging and analysis. Ford Aerospace has developed an image analysis system, SIANN, that integrates the technologies necessary to satisfy NASA's science data analysis requirements for the next generation of satellites. SIANN will enable scientists to train a neural network to recognize image data containing scenes of interest and then rapidly search data archives for all such images. The approach combines conventional image processing technology with recent advances in neural networks to provide improved classification capabilities. SIANN allows users to proceed through a four step process of image classification: filtering and enhancement, creation of neural network training data via application of feature extraction algorithms, configuring and training a neural network model, and classification of images by application of the trained neural network. A prototype experimentation testbed has been completed and applied to climatological data.
\end{abstract}

\section{INTRODUCTION}

Data acquired from satellites are essential resources in meteorology, agriculture, astronomy, forestry, geology, oceanography, and many other fields. Cataloging and analysis of image data has been a fundamental problem for NASA. For instance, in 1986 a team of scientists at the South Pole took readings overhead and learned that the "hole" in the Earth's ozone was getting worse. It was later discovered that the hole actually showed up in 1976 in Nimbus 7 satellite data. Concerning this discovery, James L. Green, head of the NASA National Space Science Data Center stated in (Kneale, 1988), "It's one of probably hundreds of important discoveries we have sitting in the basement." To compound this problem, the next generation of scientific satellites will generate far greater amounts of data.

How will such an enormous database be accessed, and how will large amounts of data be analyzed? To help provide solutions to these questions, Ford Aerospace is investigating neural network technology to determine how it can provide improved satellite image analysis capabilities. A prototype system called SIANN (Satellite Image Analysis using Neural Networks) has been developed which combines conventional image processing techniques with neural networks. Currently, SIANN addresses the image cataloging problem; that is, the generation of summary information, or "metadata", from raw image data. The metadata are stored in a database which will enable scientists to rapidly retrieve images containing scenes of interest.

SIANN is intended to be a generalpurpose classification system. It will be embedded into large satellite data management systems and provide a library of feature extraction and classification programs to support dozens of scientific disciplines.

Scientists working in different domains may be interested in the same data. As such, it is necessary to apply a variety of algorithms to the raw image data to create the metadata that will support queries from multiple scientific domains.

Scientists develop classifiers in SIANN by using the following procedure, which is illustrated in figure 1:

1) Select (or develop if necessary) feature extraction algorithms which are appropriate for the scientific 
discipline of interest and create a training set, $T$, of patterns representative of the desired classes

2) Configure and train a supervised learning neural network to identify the desired classes of image scenery using the training set $\mathrm{T}$

3) Test the classifier by applying it to novel data; if the results are not satisfactory, repeat steps 1 and/or 2 and then retest.

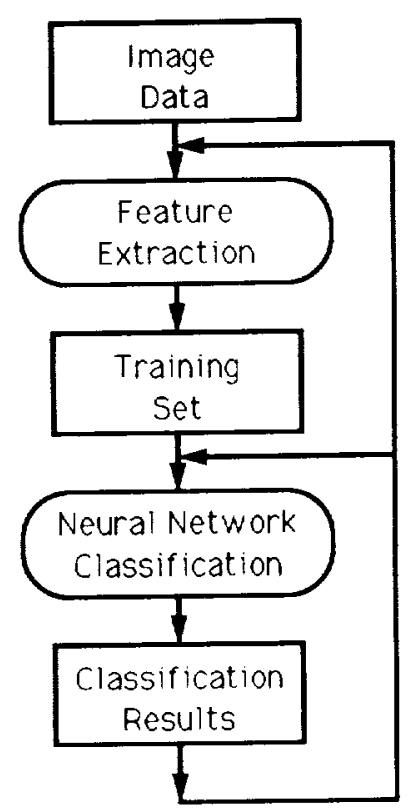

Figure 1. The SIANN image classification process. Rectangles represent data and ovals represent operations.

The creation of neural network training sets by applying feature extraction algorithms has proven successful in a number of different applications (Rimey, 1986; Beck, 1989). Another useful approach is to classify individual pixels from multispectral images (Campbell, 1989; McClellan, 1989).

This paper presents initial results of SIANN applied to climatological image data. First, the feature extraction process is described. Next, the neural network classification technique is presented. Then an experiment is described which analyzes the effects that varying the number of training set features has on a neural network's classification correctness. Finally, conclusions are made and directions of future work are stated.

\section{FEATURE EXTRACTION}

(Garand, 88) describes 13 features representing height, albedo, shape, and multilayering characteristics of cloud fields. Table 1 lists 12 of these features (Garand's feature for 'Fraction of cloudy pixels with D $<D_{\text {r }}^{\prime}$ was not included.) plus three simple statistical features and the 'Number background' feature which is a variation of the 'Number of clouds' feature.

Table 1. Features used to classify climatological data. Image source: VIS, visible; IR, infrared; B\&W, binary corresponding to visible cloud fraction; PS, power spectrum.

Description

Limits

1. Total cloud fraction (IR, VIS) $0-1$

2. Low cloud fraction (IR) 0-1

3. Middle cloud fraction (IR) $0-1$

4. High cloud fraction (IR) 0-1

5. Cloud height of uppermost layer (IR) $0-14 \mathrm{~km}$

6. Fraction of cloudy pixels (VIS) 0-1

7. Mean albedo of cloudy pixels (VIS) 0-1

8. Number of clouds (B\&W) $0-m / 2$

9. Multilayer index (IR) 0-1

10. Background connectivity (B\&W) $0-1$

11. Cloud connectivity (B\&W) $0-1$

12. Streakiness factor (PS) 0-1

13. Fraction of spectral intensity $0-1$ associated with wavelengths between $20-40 \mathrm{~km}$ (PS)

14. Minimum pixel value (VIS) 0-255

15. Maximum pixel value (VIS) 0-255

16. Range of pixel values (VIS) $\quad 0-255$

17. Number background (VIS) $0-m / 2$

Note that Garand's work is solely directed at the classification of 20 cloud types, without regard to computation time. Since classifiers created from SIANN will be applied to immense databases, there is usually a time/accuracy tradeoff. That is, computationally inexpensive features are 


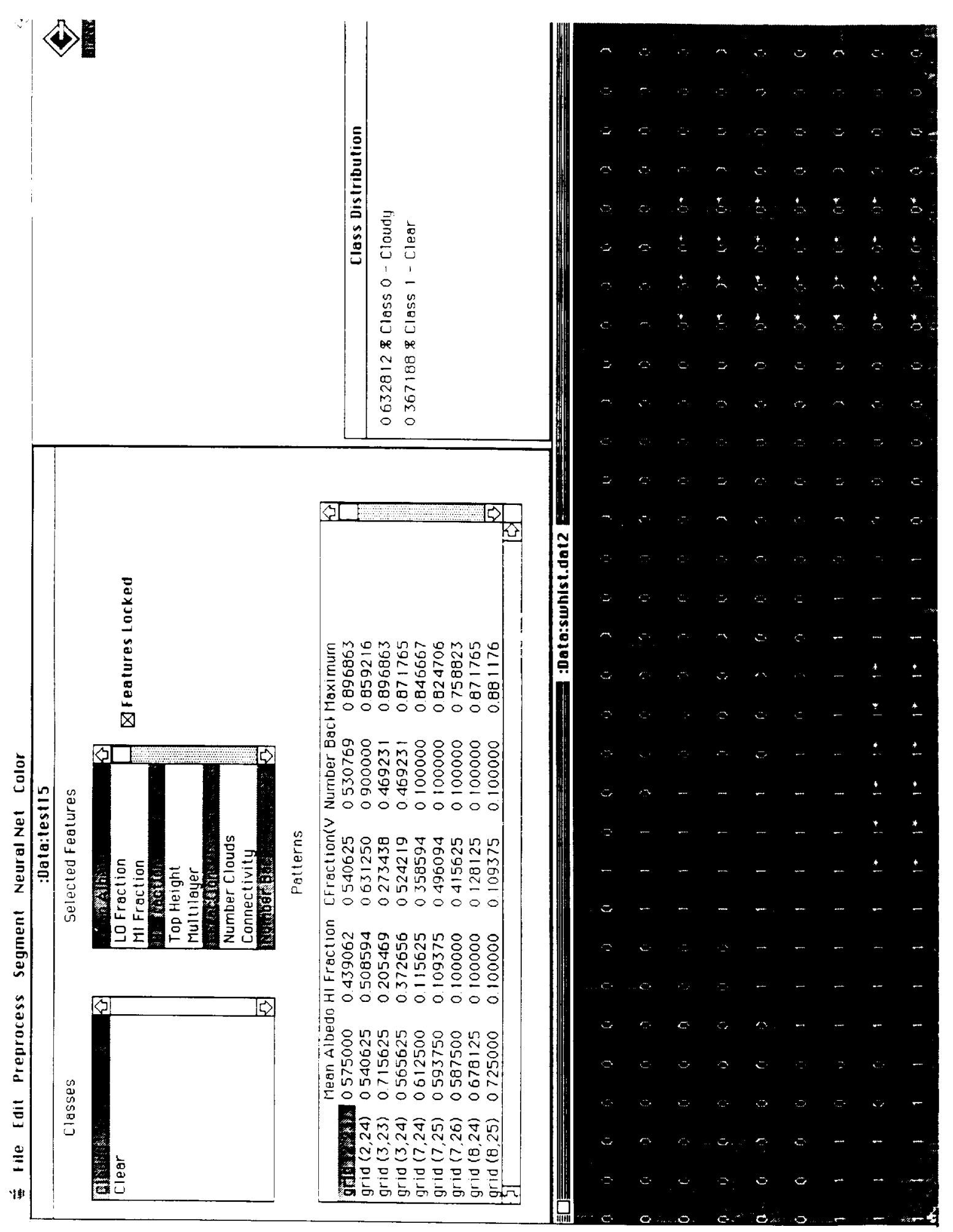

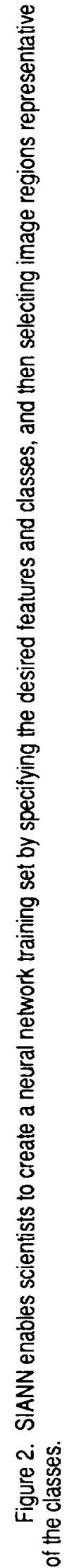


usually selected over more accurate, but more expensive features. Likewise, smaller neural networks (i.e., fewer nodes) are preferred to larger networks.

Figure 2 shows the SIANN user interface for creating a training set. The first step is to enter the desired classes of image scenes into the "Classes" box. Next, a set of features are selected from the "Features" box. Then image regions that are representative of the classes are selected as depicted by the starred ("*") regions in figure 2. Alternatively, odd sized regions may be selected in addition to the fixed-sized grid regions. Finally, a command is issued to generate the feature vectors for the selected image regions. The "Patterns" box shown in figure 2 contains the feature vectors generated from the selected image regions (but only for the currently selected class in the "Classes" box). Each feature vector consist of several real numbers typically in the range of $0 . .1$, which are used as inputs to the neural network. Each class defined by the user is represented by one output of the neural network. For the remainder of this paper, the training set shown in figure 2 shall be referred to as the "test" training set.

\section{NEURAL NETWORK CLASSIFICATION}

SIANN uses the popular backpropagation algorithm (Rumelhart, 1986). Figure 3 illustrates the general topology of a back propagation network. The bottom layer of nodes is the input layer where patterns are presented to the network. The top layer contains the output nodes which indicate the class of the input pattern. Any number of internal layers are permitted, but typically one is sufficient. (The paper by Ho, 1989 concludes that it is generally better to increase the width of the network than to add layers.)

Given a training set, SIANN will automatically configure and initialize a network. Figure 4 describes the network that SIANN generated from the test training set. (This network will be referred to as the "test" network.) Note that the number of input nodes matches the number of features and the number of output nodes matches the number of classes. Each feature value of the input patterns is scaled from the corresponding limits in table 1 to the range 0.1 to 0.9 .

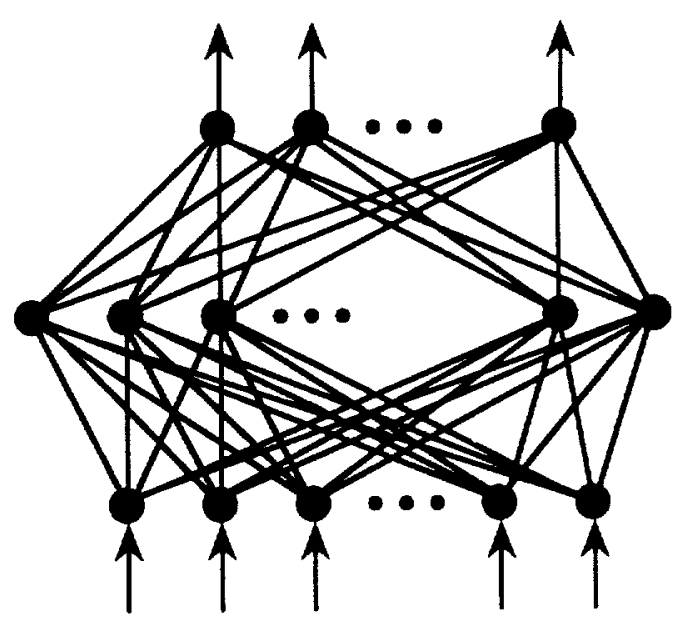

Figure 3. A back propagation neural network receives a pattern in its bottom, input layer and computes the pattern's class in the top, output layer.

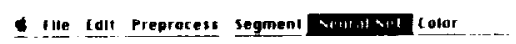

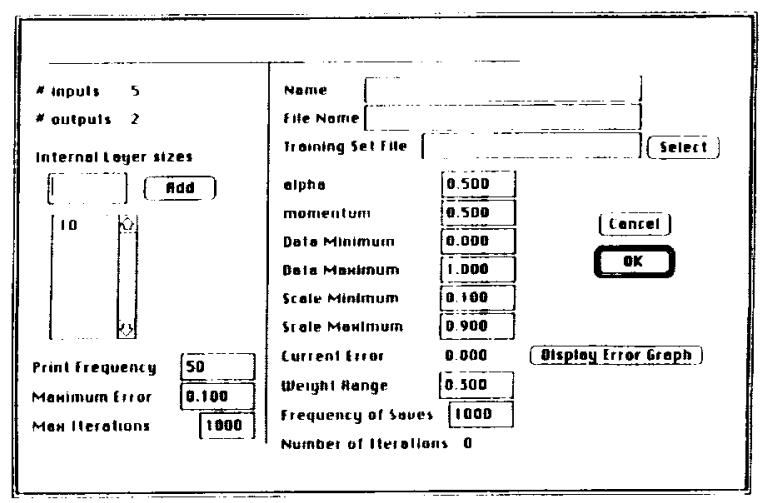

Figure 4. SIANN automatically configures a back propagation neural network to train on a specified training set.

Before training begins, SIANN automatically creates unary vectors for the target outputs. For the 2-class test network, the vectors are:

$\begin{array}{lll}\text { Class 0: } & 0.1 & 0.9 \\ \text { Class 1: } & 0.9 & 0.1\end{array}$

After training, the network is tested by applying it to new data (i.e., data that it 
wasn't trained with). Figure 5 shows the results of classifying the test image with the test network. Misclassifications are denoted by an "X $i$ " where $\mathrm{X}$ means WRONG, and $i$ is the class computed by the network. If the network does not perform satisfactorily, the scientist may modify the training set and/or network and then retest.

\section{EXPERIMENTAL RESULTS}

The data set used contains three $1024 \mathrm{x}$ 3848 bit AVHRR (Advanced Very High Resolution Radiometer) images of the Indian Ocean. Each 8 bit pixel has a footprint of 1 $\mathrm{km}^{2}$. For purposes of this discussion, we shall focus on the image shown in figure 2 , which has been overlaid with the author's subjective classification of the picture. (This image will be referred to as the "test" image.)

Table 2. Six training sets were created containing from 4 to 9 features.

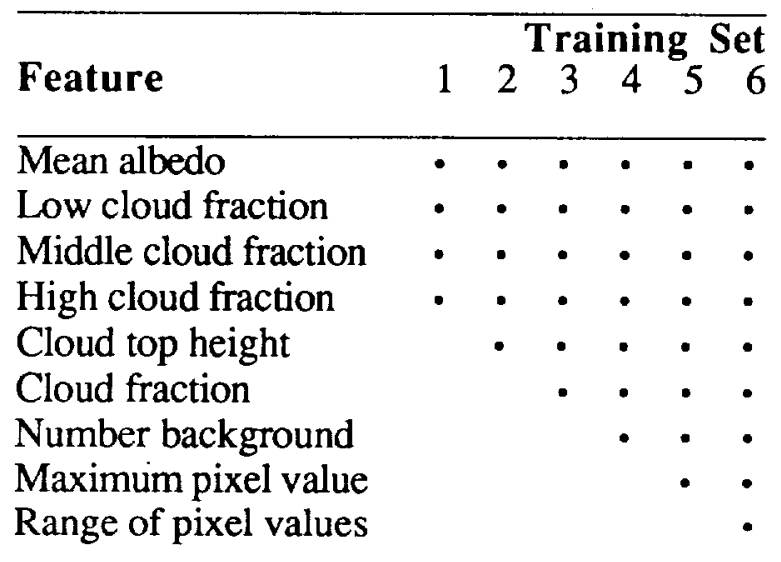

An experiment was conducted to determine the effects of modifying the set of selected features. Six 2-class training sets were created, each containing patterns from the size $32^{2}$ grid regions lying in the rectangle whose upper left grid coordinates are $(4,10)$ and lower right grid coordinates are $(7,20)$. This provided 25 Cloudy patterns and 19 Clear patterns for each training set. The features used in each training set are listed in table 2. Each training set was used to train a network. Each network had a single internal layer of 15 nodes. Equation (1) was used during training to modify each weight, where the learning rate $\alpha=0.9$, and the momentum term $\eta=0.7$.

$$
\begin{aligned}
w_{i j}(t+1)= & w_{i j}(t)+ \\
& \eta \delta_{j} x_{j}+\alpha\left(w_{i j}(t)-w_{i j}(t-1)\right)
\end{aligned}
$$

Convergence for each network occurred when the maximum error fell below 0.1 . Table 3 summarizes the results of training the networks. The third column specifies how many training iterations each network required to converge. The fourth column lists the CPU time of each training run on a VAXstation 3540. Note that when the number of features decrease, the time for each iteration also decreases since the number of nodes in the network is reduced.

Table 3. Training times increase as the number of input features decreases.

\begin{tabular}{llll}
\hline $\begin{array}{l}\text { Training } \\
\text { Set }\end{array}$ & $\begin{array}{l}\text { Number } \\
\text { Features }\end{array}$ & $\begin{array}{l}\text { Number } \\
\text { Iterations }\end{array}$ & Minutes \\
\hline 1 & 4 & 1790 & 5.6 \\
2 & 5 & 2278 & 7.8 \\
3 & 6 & 711 & 2.7 \\
4 & 7 & 351 & 1.5 \\
5 & 8 & 316 & 1.3 \\
6 & 9 & 456 & 2.1 \\
\hline
\end{tabular}

The nonlinearity of the number of iterations and minutes for training runs can be attributed to the characteristics of certain features. Specifically, it would appear that the addition of the 'Cloud top height' feature that distinguishes training set 1 from training set 2 detracts from the separability of patterns within training set 2 . Similarly, the addition of the 'Range of pixel values' feature detracts from the separability of training set 6 .

Each network was tested by applying it to all 384 grid regions of the test image. Figure 6 plots the percentage of misclassified regions vs. the number of features. The number of classification errors tends to decrease when a larger number of input features are used. 


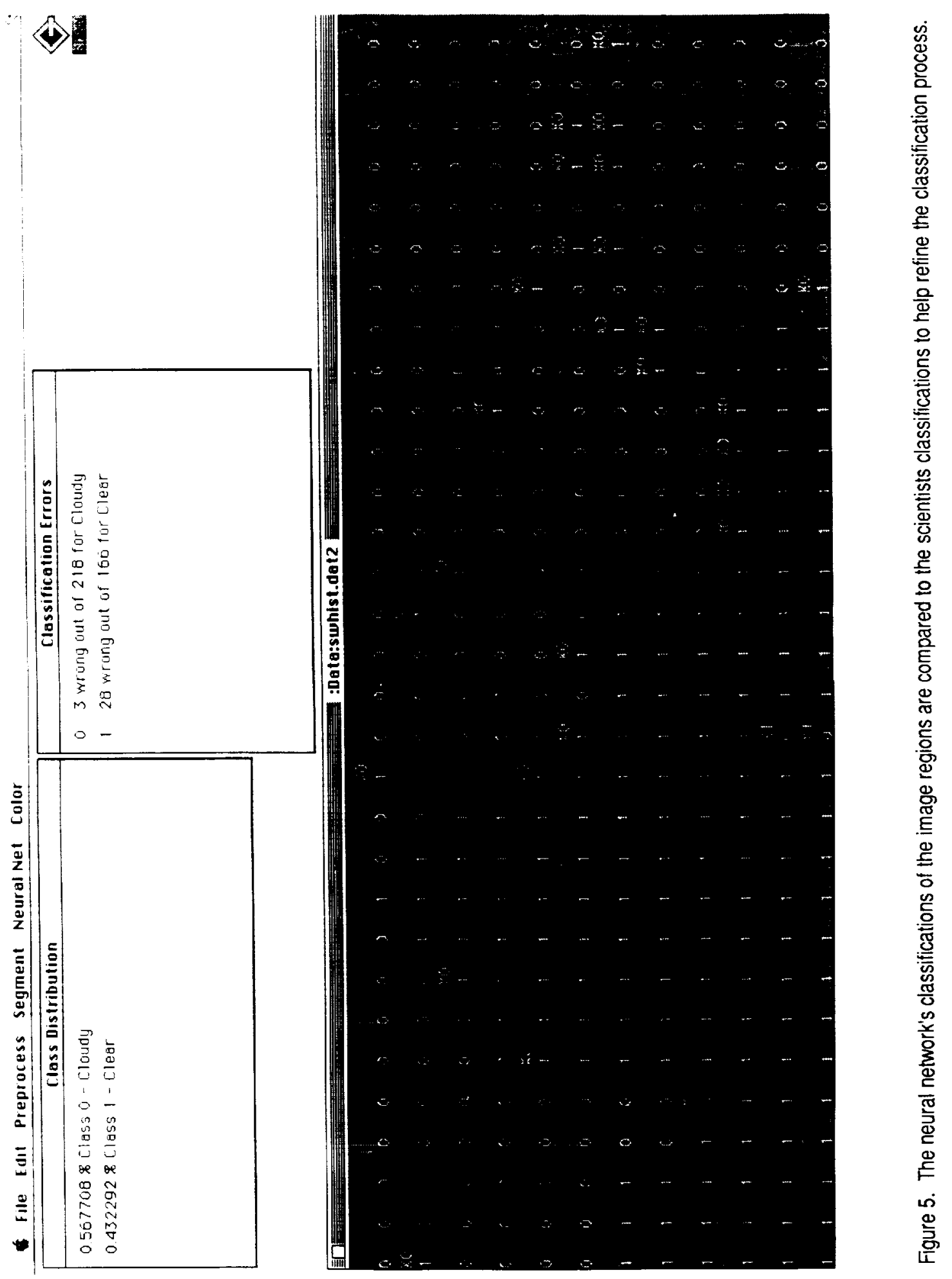




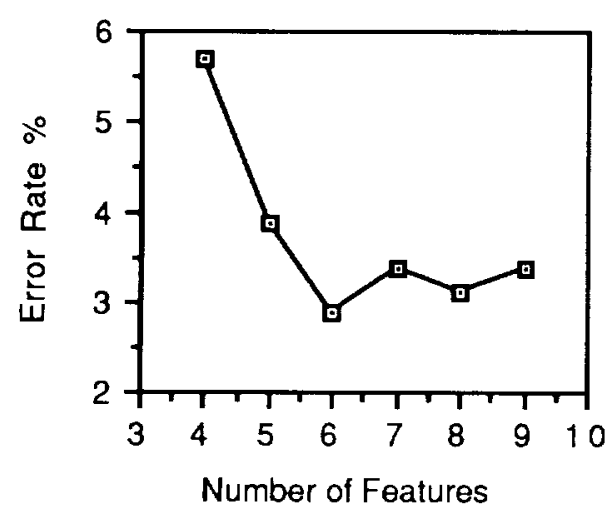

Figure 6. Neural network classification errors tend to increase as the number of input features decreases.

Scientists using SIANN can analyze training and classification results to select the optimal set of features and most efficient neural network configuration.

\section{CONCLUSIONS}

SIANN is an image analysis system that combines conventional image processing techniques with neural network classifiers. Scientists may quickly develop a customized classifier using SIANN's menu-driven graphical interface. Analysis of the classifier's behavior helps the scientist improve the classifier by modifying the training set features and neural network configuration.

Preliminary testing of the system on climatological data has demonstrated that neural networks are a viable technique for image analysis. SIANN will continue to evolve by adding feature extraction programs for other scientific domains. Another future direction is to investigate unsupervised learning neural networks to determine if the classifier refinement process can be automated; that is, to see if a network can discover by itself a good set of features.

\section{REFERENCES}

Beck, H., McDonald, D., \& Brzakovic, D. (1989). A self-training visual inspection system with a neural network classifier. Proceedings from the 1989 International
Joint Conference on Neural Networks (pp. I-307 - I-311). Washington, D.C.

Campbell, W., Hill, S., \& Cromp, R. (1989). The utilization of neural nets in populating an object-oriented database. Proceedings from the 1989 Goddard Conference on Space Applications of Artificial Intelligence. (pp. 249-263). Greenbelt, MD.

Garand, L. (1988). Automated recognition of oceanic cloud patterns. Part I: Methodology and application to cloud climatology. Journal of Climate, 1(1), 20-39.

Ho, C. (1989). On multi-layered connectionist models: Adding layers vs. increasing width. Proceedings from the Eleventh International Joint Conference on Artificial Intelligence. (pp. 176-179). Washington, D.C.

Kneale, D. (January 11, 1988). What becomes of data sent back from space? Not a lot, as a rule. Wall Street Journal, p. 1.

McClellan, G., DeWitt, R., Hemmer, T., Matheson, L., \& Moe, G. (1989). Multispectral image-processing with a threelayer backpropagation network. Proceedings from the 1989 International Joint Conference on Neural Networks (pp. I-151 - I-153). Washington, D.C.

Rimey, R., Gouin, P., Scofield, C., \& Reilly, D. (1986). Real-time 3-D object classification using a learning system. SPIE Intelligent Robots: 6th International Conference on Robot Vision and Sensory Controls, RoViSeC, SPIE Vol. 726. (pp. 552-557). Cambridge, MA.

Rumelhart, D., \& McClelland, J. (1986). Parallel Distributed Processing: Explorations in the Microstructure of Cognition. Cambridge, MA: MIT Press. 
- 


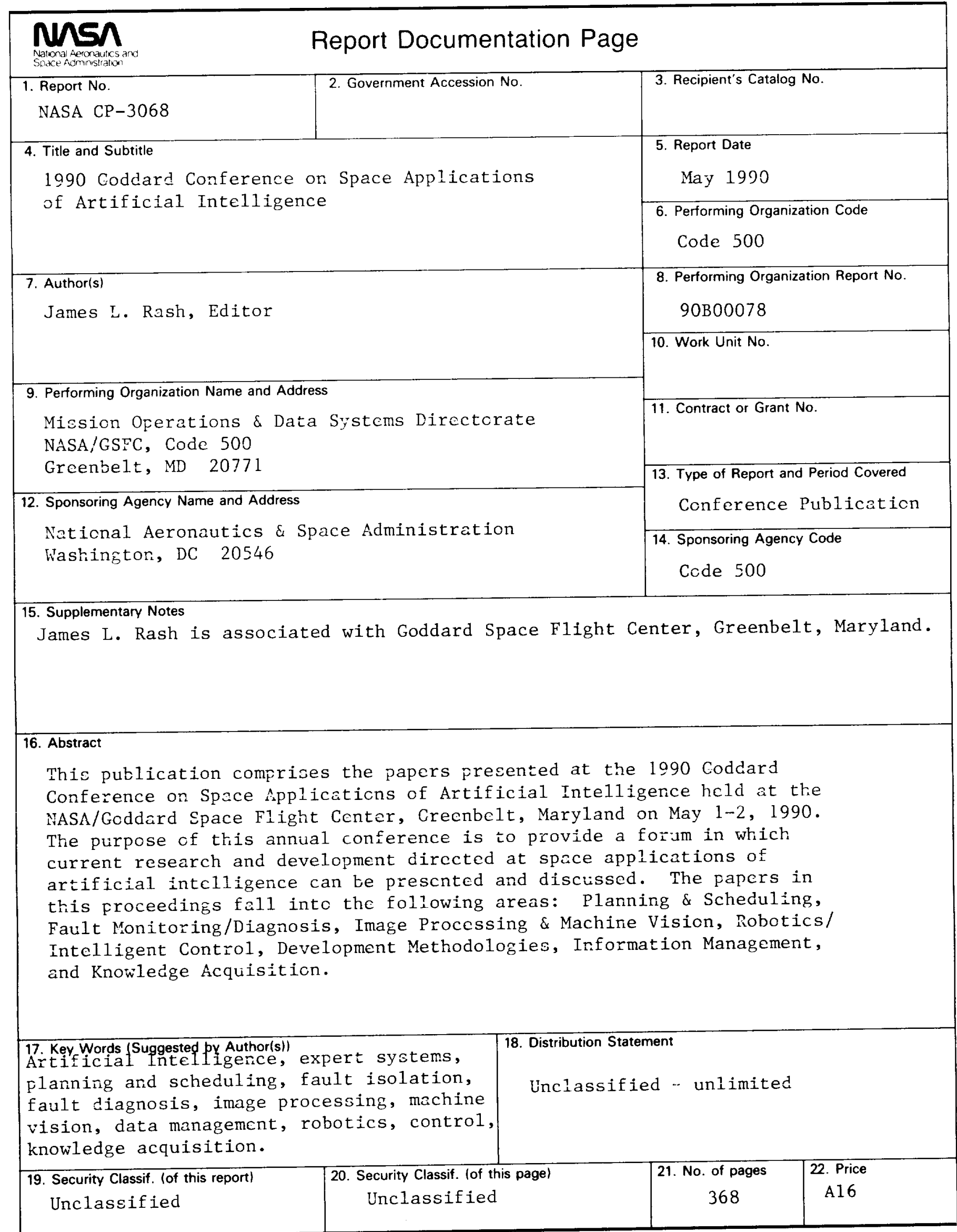


